\title{
Monocular Diplopia
}

National Cancer Institute

\section{Source}

National Cancer Institute. Monocular Diplopia. NCI Thesaurus. Code C118719.

Perception of more than one image when viewing with one eye. 